\title{
Optimization of Potato Flavor Yoghurt by Response Surface Method
}

\author{
Li Cai ${ }^{\text {a }, ~ G u o c h a o ~ G a n, ~ Q i a o ~ L i n ~}{ }^{*}$, Fayong Gong, Zhong Zhang, Lijun He \\ Xichang University of Agricultural Science, Xichang 615013, Sichuan \\ *Corresponding author Email: 66656736@qq.com \\ a5696132@qq.comm,
}

Keywords: Potato Flavor of Yogurt, Quality, Response Surface Method.

\begin{abstract}
In this experiment, potato leben-amylase hydrolysate and fresh milk were used as the main raw materials to make potato yoghurt. On the basis of five single factor experiments, including the ratio of feed to liquid, the amount of sugar added, the amount of fermentation agent added, the fermentation temperature and the fermentation time, the fermentation process of potato flavor yogurt was optimized by selecting the ratio of feed to liquid, the amount of sugar added and the amount of fermentation agent added as the main influencing factors. The results showed that the best quality of yogurt was obtained when the ratio of remelted potato leuben-amylase lysate to fresh milk was $1: 1$, the adding amount of granulated sugar was $8 \%$, and the adding amount of leavening agent was $0.6 \%$.
\end{abstract}

\section{Introduction}

Since 2015, China has launched the strategy of potato staple food, and the country has vigorously promoted potato processing, providing policy guidance for potato deep processing. Potato is the main high-efficiency dominant crop in Liangshan Prefecture. Developing potato processing products is of great significance to accelerate the industrialization of potato planting agriculture in Liangshan Prefecture [1]. The purpose of this experiment is to produce potato flavor yoghurt with excellent quality by compound fermentation of potato enzymatic hydrolysate and milk.

\section{Materials and methods}

\subsection{Test materials and instruments}

\subsubsection{Materials}

Fresh potato: Qingshu 9; High temperature resistant $\alpha$-amylase: Hunan Hongyingxiang Bioengineering Co., Ltd., with activity of 30000 U/g; Lysell pure milk and soft white sugar: sold at Walmart supermarket in Xichang; Starter: Bai Sheng You yogurt fermented bacteria powder.

\subsubsection{Instruments}

Electronic balance, induction cooker, colloid mill, constant temperature and humidity incubator, full-automatic Kjeldahl nitrogen determinator.

\section{Experimental design}

\subsubsection{Technological process}

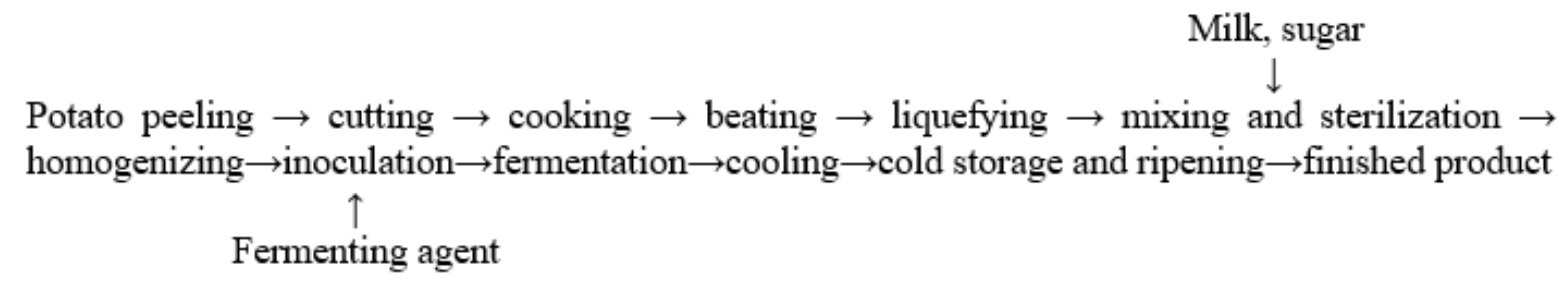




\subsubsection{Key points of technology}

(1) Liquefaction: The addition amount of $\alpha$-amylase is $0.4 \%$ of the amount of cooked potato juice, which is liquefied at normal temperature for $30 \mathrm{~min}$ to obtain cooked potato $\alpha$-amylase enzymatic hydrolysate for later use.

(2) blending and sterilization: the cooked potato enzymolysis liquid, pure milk and soft white sugar are weighed and blended according to a certain proportion, after being evenly mixed, sterilization treatment is carried out under the conditions of 100 DEG C and 15min [2-4].

(3) Homogenization: Homogenizing the mixture of milk, cooked potato enzymatic hydrolysate and sugar with colloid mill for $10 \mathrm{~min}$.

(4) Inoculation: the homogenized mixed solution is rapidly cooled, yogurt starter is rapidly inoculated, stirred evenly and then split into plastic cups and sealed with plastic wrap.

(5) Fermentation culture: put the inoculated homogenate into the fermentation tank and ferment at $42{ }^{\circ} \mathrm{C}$ for $4 \mathrm{~h}$, take it out for cooling, transfer it to the refrigerator and refrigerate it for ripening.

\subsubsection{Single factor test}

\subsubsection{Single factor test of the ratio of cooked potato $\alpha$-amylase digestion solution to pure milk}

The ratio of cooked potato $\alpha$-amylase enzymolysis liquid to pure milk is 3:7, 2:3, 1:1, 3:2 and 7:3 respectively. The addition of soft sugar is $8 \%$ and $0.2 \%$ leavening agent is added, and the soft sugar is placed in an incubator at 42 DEG C for fermentation for 4 hours, then refrigerated and cooked. Protein content was determined and sensory scores were performed. Three groups of parallel experiments were conducted for each material-liquid ratio. Based on the protein content of yoghurt products, the sensory evaluation index was used for data processing to establish the best solid-liquid ratio.

\subsubsection{Single factor experiment on addition amount of soft sugar}

Under the condition that the ratio of potato enzymolysis liquid to pure milk is $1: 1,2 \%, 4 \%, 6 \%$, $8 \%$ and $10 \%$ soft sugar are added respectively. Then, $0.2 \%$ starter was added, fermented at $42^{\circ} \mathrm{C}$ for 4 hours and then taken out of the fermentation box, and put into the refrigerator for refrigeration and ripening. The effect of soft sugar addition on yogurt fermentation was studied.

\subsubsection{Single factor experiment of different adding amount of starter}

Under the condition that the ratio of cooked potato enzymolysis liquid to pure milk is $1: 1$ and the addition amount of soft sugar is $8 \%$, inoculation fermentation is carried out according to the inoculation amounts of $0.2 \%, 0.4 \%, 0.6 \%, 0.8 \%$ and $1.0 \%$. After fermentation at $42^{\circ} \mathrm{C}$ for 4 hours, it was taken out of the fermentation box and put into the refrigerator for cold storage and ripening. Each experiment was repeated 3 times to study the effect of the amount of inoculum on yogurt fermentation.

\subsubsection{Single factor experiment of different fermentation time}

The ratio of cooked potato hydrolysate to pure milk was $1: 1$, and the addition of soft sugar was $8 \%$. Under the condition of $6 \%$ inoculum, the yogurt was fermented at $42^{\circ} \mathrm{C}$ for $3 \mathrm{~h}, 4 \mathrm{~h}, 5 \mathrm{~h}, 6 \mathrm{~h}$ and $7 \mathrm{~h}$ respectively, then refrigerated and cooked. Each experiment was repeated for 3 times to study the effect of fermentation time on yogurt fermentation.

\subsubsection{Single factor experiment of different fermentation temperature}

The ratio of cooked potato hydrolysate to pure milk was $1: 1$, and the addition of soft sugar was $8 \%$. Under the condition that the inoculation amount is $6 \%$, it is fermented at $24^{\circ} \mathrm{C}, 30^{\circ} \mathrm{C}, 36^{\circ} \mathrm{C}$, $42^{\circ} \mathrm{C}$ and $48^{\circ} \mathrm{C}$ for 4 hours and then refrigerated and cooked. Each test is repeated 3 times. The effect of fermentation temperature on yogurt fermentation was studied. 


\subsubsection{Response surface method for optimal design}

Based on the single-factor experimental design, this experimental design selects the ratio of solid to liquid x1, the addition amount of soft sugar x2, and the addition amount of starter x3. These three factors are independent variables, and a response surface optimization test scheme with three factors and three levels is designed. With sensory evaluation score as dependent variable $\mathrm{Y}$, response surface analysis is carried out on the process of potato flavor fermented yoghurt by using Design-Expert 8.0.5 software and Box-Behnken test design principle, and the optimal process optimization parameters [5-7] are determined. The test factor levels are shown in Table 1.

Table 1 Factor Level

\begin{tabular}{|c|c|c|c|}
\hline Factor & & Coding level & \\
\cline { 2 - 3 } & -1 & 0 & 1 \\
\hline A material-liquid ratio & $2 / 3$ & $1 / 1$ & $3 / 2$ \\
\hline B addition amount of soft white sugar/\% & 6 & 8 & 10 \\
\hline C starter addition/\% & 0.4 & 0.6 & 0.8 \\
\hline
\end{tabular}

\subsection{Evaluating indicator}

\subsubsection{Sensory evaluation}

The sensory evaluation standard of potato flavor yoghurt is formulated with reference to GB 19302-2010. The sensory evaluation is conducted on the test results from four aspects: color, taste, smell and tissue state. The evaluation standard is shown in Table 2.

Table 2 Potato Flavor of Yogurt Evaluation Table

\begin{tabular}{|c|c|c|}
\hline & Scoring criteria & Score \\
\hline \multirow{4}{*}{$\begin{array}{l}\text { Colour and lustre } \\
\text { (25) }\end{array}$} & Uniform color, milky white or yellowish & $20-25$ \\
\hline & Uniform color, light yellow & $15-20$ \\
\hline & $\begin{array}{l}\text { Uneven color distribution, poor luster, dark } \\
\text { yellow }\end{array}$ & $10-15$ \\
\hline & Uneven color distribution and turbidity & $<10$ \\
\hline \multirow{4}{*}{$\begin{array}{c}\text { Smell } \\
(25)\end{array}$} & With potato fragrance and yogurt flavor & $20-25$ \\
\hline & $\begin{array}{l}\text { Has a light potato fragrance and yogurt } \\
\text { flavor }\end{array}$ & $15-20$ \\
\hline & $\begin{array}{c}\text { The aroma of potato and yoghurt is not } \\
\text { obvious }\end{array}$ & $10-15$ \\
\hline & $\begin{array}{l}\text { No potato fragrance and sour milk } \\
\text { fragrance, with peculiar smell }\end{array}$ & $<10$ \\
\hline \multirow{4}{*}{$\begin{array}{l}\text { Taste } \\
(25)\end{array}$} & Potato flavor, moderate sweet and sour & $20-25$ \\
\hline & Slightly potato flavor & $15-20$ \\
\hline & $\begin{array}{c}\text { Potato flavor is not obvious, the taste is too } \\
\text { sour or too sweet }\end{array}$ & $10-15$ \\
\hline & No potato flavor, bad taste & $<10$ \\
\hline \multirow{4}{*}{$\begin{array}{l}\text { Organization } \\
\text { condition } \\
\text { (25) }\end{array}$} & $\begin{array}{l}\text { Fine and even structure, no whey } \\
\text { precipitation }\end{array}$ & $20-25$ \\
\hline & $\begin{array}{l}\text { Fine and even structure with a small } \\
\text { amount of whey }\end{array}$ & $15-20$ \\
\hline & $\begin{array}{l}\text { The structure is fine and even with whey } \\
\text { precipitation }\end{array}$ & $10-15$ \\
\hline & $\begin{array}{l}\text { There is a large amount of whey } \\
\text { precipitation with precipitation }\end{array}$ & $<10$ \\
\hline
\end{tabular}




\subsubsection{Protein content}

The determination method of protein content in tartary buckwheat seeds refers to GB5009.5-2016.

\section{Results and analysis}

\section{Single factor test results}

3.1.1 Single factor test results of the ratio of enzymatic hydrolysate of mature potato to pure milk

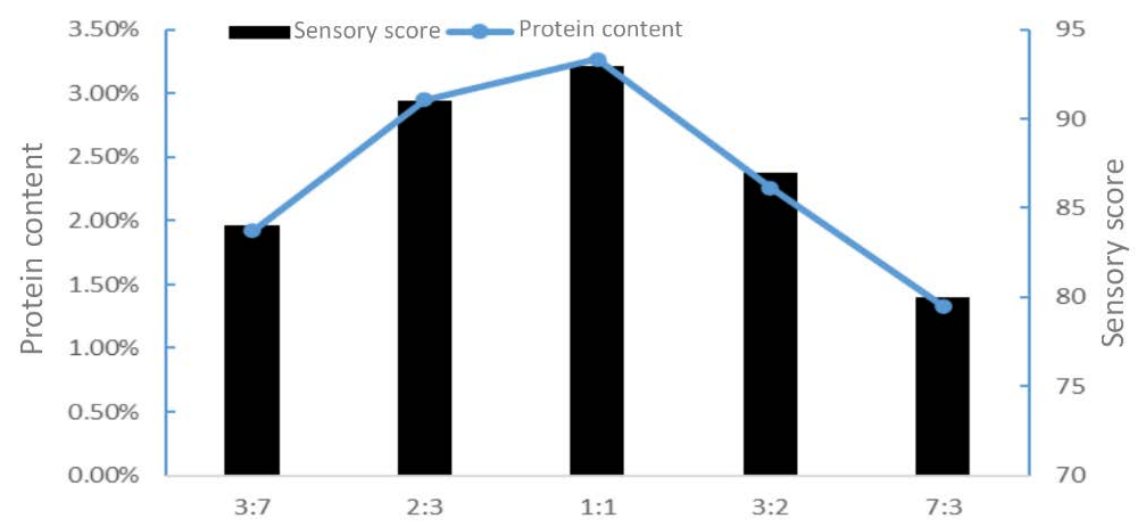

Figure 1 The effect of the ratio of enzymolysis solution to milk on the quality of yogurt

As can be seen from fig. 1, when the ratio of cooked potato enzymatic hydrolysate to pure milk is $7: 3$, the protein content of potato flavor yoghurt is obviously lower than the national standard requirements, and the sensory evaluation score is also lower. When the ratio is $1: 1$, the potato flavor yogurt has the best protein content and sensory evaluation.

\subsubsection{Single factor test results of the amount of soft white sugar added}

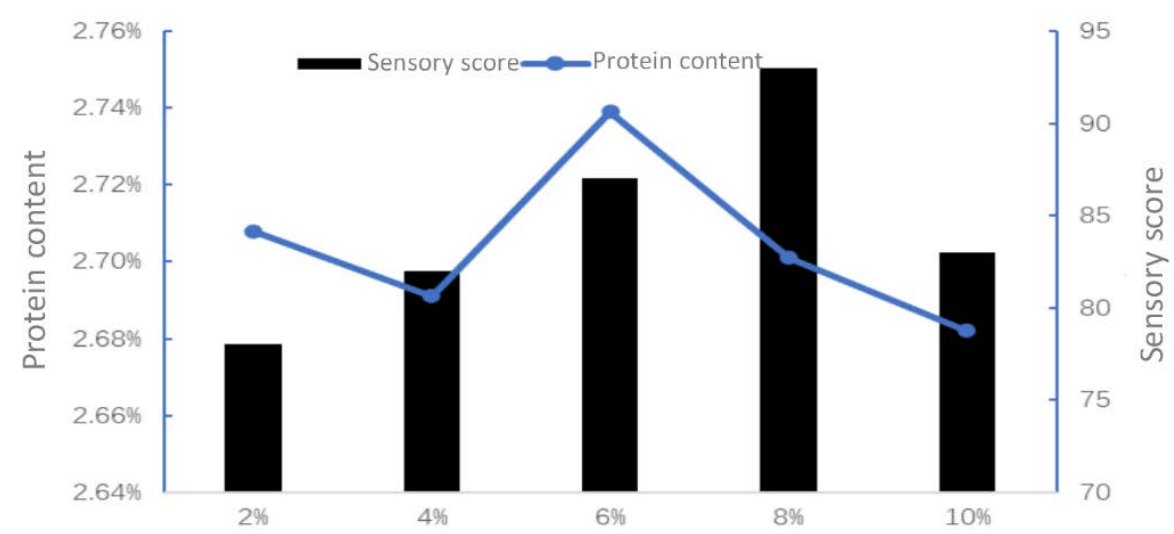

Figure 2 The effect of the addition of cotton sugar on the quality of yogurt

As can be seen from fig. 2, each sugar content has no obvious influence on the protein content, and there is no obvious correlation between sugar content and protein. When the proportion of soft sugar is $8 \%$, the sensory evaluation score of potato flavor yoghurt is the highest. When the sugar content is $4 \%, 6 \%$ and $8 \%$, the comprehensive evaluation of potato flavor yoghurt is better. 


\subsubsection{Single factor test results of the added amount of starter}

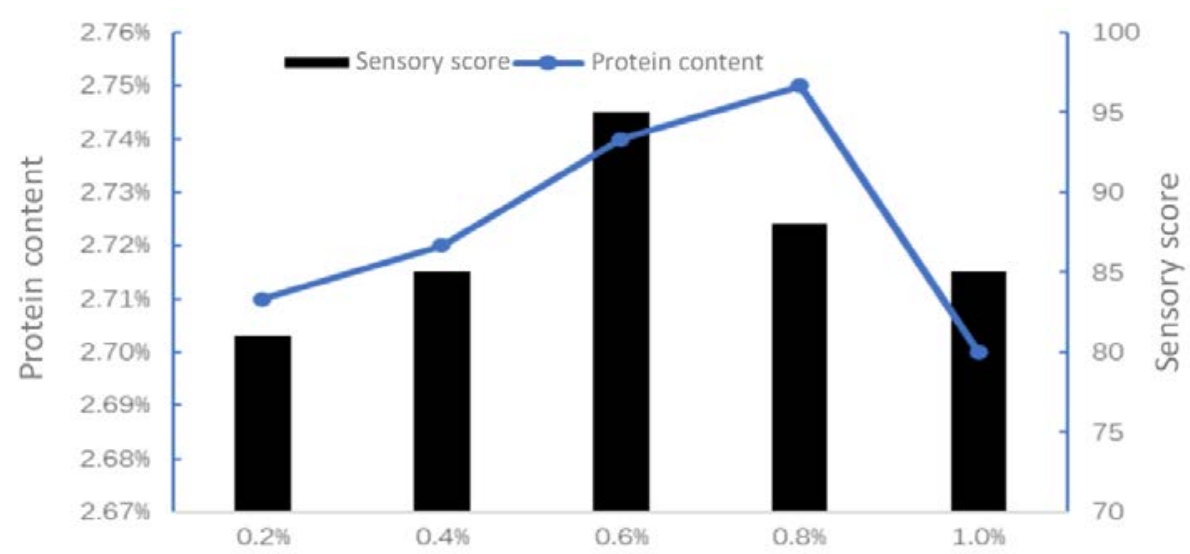

Figure 3 The effect of the amount of starter on the quality of yogurt

As can be seen from fig. 3, when the inoculation amount of the starter is $0.6 \%$, the sensory evaluation score of potato flavor yogurt is the highest. The comprehensive evaluation of potato flavor yoghurt is better when the inoculum size of starter is $0.4 \%, 0.6 \%$ and $0.8 \%$.

\subsubsection{Single factor test results of fermentation time}

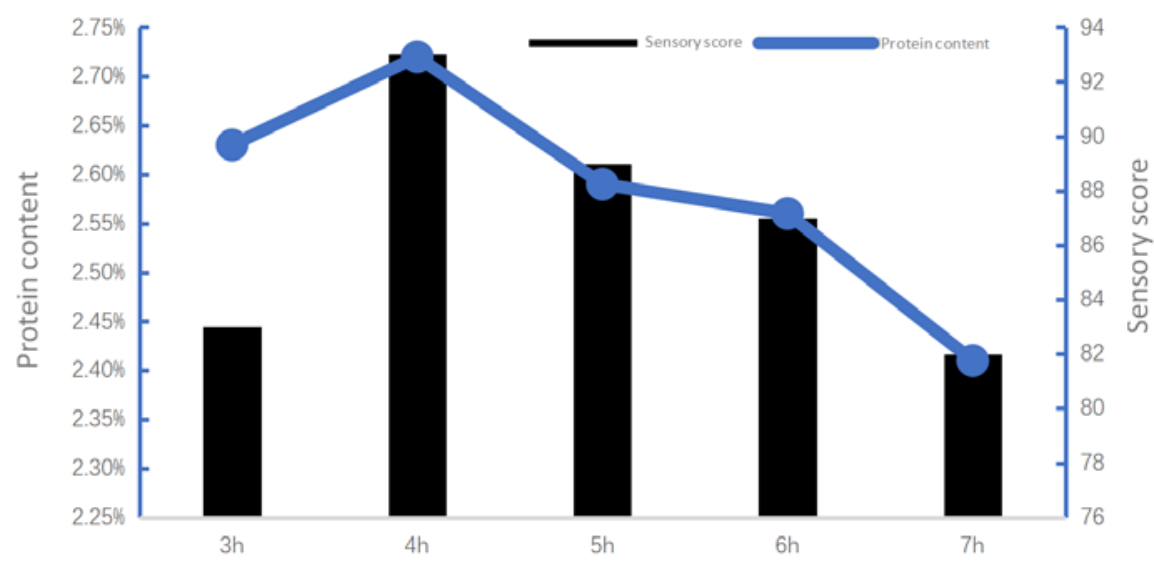

Figure 4 The effect of fermentation time on the quality of yogurt

As can be seen from fig. 4, when the fermentation time is $7 \mathrm{~h}$, the protein content obviously decreases, and the fermentation effect is the worst at this time, which may be due to excessive fermentation and obvious hydrolysis of casein curd in yogurt. When the fermentation time is $4 \mathrm{~h}, 5 \mathrm{~h}$ and $6 \mathrm{~h}$, the comprehensive evaluation of potato flavor yoghurt is better, especially $4 \mathrm{~h}$.

\subsubsection{Single factor test results of fermentation temperature}

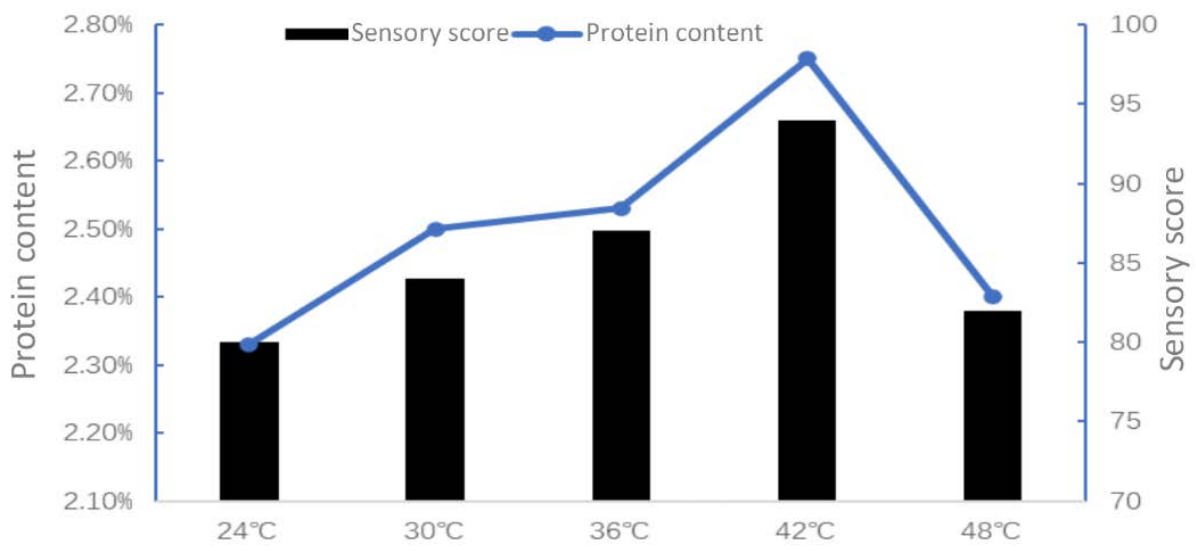

Figure 5 Effect of fermentation temperature on yogurt quality 
As can be seen from fig. 5, when the fermentation temperature is $30^{\circ} \mathrm{C}, 36^{\circ} \mathrm{C}$ and $42^{\circ} \mathrm{C}$, the comprehensive evaluation of potato flavor yoghurt is better. When the fermentation temperature was $42^{\circ} \mathrm{C}$, the sensory evaluation score and protein content of potato flavor yoghurt were the highest.

\subsection{Response surface test results}

Table 3 Box-Behnken combination test results

\begin{tabular}{cccccc}
\hline Test number & $\mathrm{A}$ & $\mathrm{B}$ & $\mathrm{C}$ & Protein content & Sensory score \\
\hline 1 & 50.00 & 0.60 & 8.00 & 3.1850 & 92.3 \\
2 & 40.00 & 0.40 & 6.00 & 2.5386 & 83.5 \\
3 & 40.00 & 0.80 & 10.00 & 2.7131 & 80.5 \\
4 & 60.00 & 0.80 & 6.00 & 2.3260 & 80.2 \\
5 & 50.00 & 0.60 & 8.00 & 3.1890 & 91.2 \\
6 & 60.00 & 0.40 & 6.00 & 2.3150 & 89.8 \\
7 & 40.00 & 0.40 & 10.00 & 2.9842 & 88.6 \\
8 & 50.00 & 0.26 & 8.00 & 2.8197 & 87.9 \\
9 & 50.00 & 0.94 & 8.00 & 2.7324 & 95.6 \\
10 & 50.00 & 0.60 & 8.00 & 3.2000 & 80.2 \\
11 & 50.00 & 0.60 & 11.36 & 3.1970 & 81.2 \\
12 & 50.00 & 0.60 & 4.64 & 3.2130 & 84.5 \\
13 & 33.18 & 0.60 & 8.00 & 3.0160 & 91.8 \\
14 & 60.00 & 0.40 & 10.00 & 2.3790 & 83.1 \\
15 & 50.00 & 0.60 & 8.00 & 3.2134 & 90.5 \\
16 & 60.00 & 0.80 & 10.00 & 2.3330 & 91.0 \\
17 & 50.00 & 0.60 & 8.00 & 3.1980 & 84.3 \\
18 & 50.00 & 0.60 & 8.00 & 3.1870 & 80.5 \\
19 & 40.00 & 0.80 & 6.00 & 3.1117 & \\
20 & 66.82 & 0.60 & 8.00 & 2.1118 & \\
\hline
\end{tabular}

Table 4 Analysis of Variance

\begin{tabular}{ccccccc}
\hline Source & The sum & $\begin{array}{c}\text { Degrees of } \\
\text { freedom df }\end{array}$ & $\begin{array}{c}\text { Mean } \\
\text { square }\end{array}$ & F value & P > F & Distinctiveness \\
\hline $\begin{array}{c}\text { Model } \\
\text { A-material to }\end{array}$ & 106.78 & 9 & 48.850 & 31.580 & $<0.0001$ & $* * *$ \\
liquid ratio & 12.22 & 1 & 3.360 & 59.000 & $<0.0001$ & $* * *$ \\
B-vaccination & 12.20 & 1 & 0.230 & 6.840 & 0.0258 & $*$ \\
C-sugar & 112.29 & 1 & 6.020 & 5.650 & $<0.0001$ & $* * *$ \\
AB & 0.045 & 1 & 0.045 & 0.025 & 0.8769 & \\
AC & 18.00 & 1 & 18.000 & 10.090 & 0.0099 & $* *$ \\
BC & 3.13 & 1 & 3.130 & 1.750 & 0.2150 & $*$ ** \\
A^2 & 221.83 & 1 & 221.830 & 124.400 & $<0.0001$ & $* *$ \\
B^2 & 24.62 & 1 & 24.620 & 13.810 & 0.0040 & $* * *$ \\
C^2 & 299.63 & 1 & 299.630 & 168.020 & $<0.0001$ & \\
Residual & 17.83 & 10 & 1.780 & & & \\
Mismatch & 0.88 & 5 & 0.180 & 0.0520 & 0.9972 & \\
Net error & 16.95 & 5 & 3.39 & & & \\
Total & 524.62 & 19 & & & & \\
deviation & & & & & \\
\hline
\end{tabular}


Note [16]:; *** is extremely significant $(\mathrm{P}<0.001)$, ** is highly significant $(\mathrm{P}<0.01)$; * is significantly different $(\mathrm{P}<0.05)$

\begin{tabular}{cccc}
\hline Std. Dev. & 1.34 & R-Squared & 0.9660 \\
\hline Mean & 85.38 & Adj R-Squared & 0.9354 \\
C.V. \% & 1.56 & Pred R-Squared & 0.9400 \\
PRESS & 31.45 & Adeq Precision & 14.580 \\
\hline
\end{tabular}

\subsubsection{Analysis results of significance test}

It can be seen from Table 3 that the model $\mathrm{p}<0.0001<0.05$ indicates that this model fits well with the actual situation and is extremely significant. The lack-of-fit term $p=0.9972 \square 0.05$, the lack-of-fit term is not significant, indicating that the test model can be better fitted with the sensory situation of potato flavored yogurt, and the test error is smaller. Therefore, this model can be used to analyze the experimental data and predict the response value. The effects of the feed-liquid ratio (A), the inoculum of bacteria (B), and the amount of added sugar (C) in the first term of the model are significant; in the second term, the inoculum of bacteria (B) reaches a highly significant level , The material-liquid ratio (A) and the amount of sugar added (C) reached a very significant level; in the interaction term, the influence of AC reached a highly significant level.

The determination coefficient R2 of the model is $96.6 \%$, which indicates that the model can explain the change of $96.6 \%$ response value, and $93.54 \%$ after correction, which indicates that the equation fits well and the coefficient of variation is low, which is 1.56 , indicating that the test has high accuracy and strong reliability.

After excluding insignificant variables, the multiple linear regression equation between the potato-flavored yoghurt to feed ratio, the amount of starter inoculum, and the amount of cotton white sugar added is:

Sensory score $\mathrm{Y}=-66.61+3.27 * \mathrm{~A}+48.45 * \mathrm{~B}+15.69 * \mathrm{C}+0.075 *$ A * C-0.039* A2-32.68* B2-1.14* C2

Wherein: a: the ratio of cooked potato enzymolysis liquid to pure milk, b: the inoculation amount of the starter, c: the addition amount of soft sugar. The number of values in front of each term in the equation can indicate the degree to which each factor affects the response value, while the values+,-represent the direction to affect the response value,+is positive and-is negative.

The combination of various factors that can obtain the highest value by using the extreme value method of compound function is: A:49.66\% B:0.58\% C:8.12\%, and the theoretical value of sensory score is 92.0983 .

\subsubsection{Response surface analysis of interaction between two factors}

The interactive effects of three factors on sensory evaluation of potato flavor yogurt can be analyzed by analyzing the regression linear equation. The interactive effects of several factors on sensory evaluation can be clearly seen in the 3D images and contour maps drawn by Desigan-Expert software.
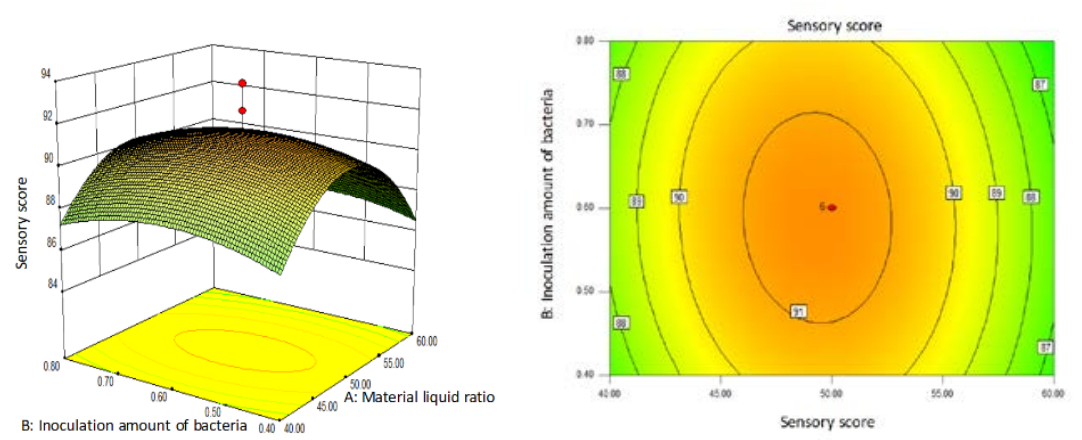

Figure 6 The interaction between the ratio of cooked potato enzymolysis solution and pure milk and the inoculation amount of starter 
As can be seen from fig. 6, with the ratio of cooked potato enzymatic hydrolysate to pure milk and the gradual increase of inoculum size, the sensory score shows a trend of increasing first and then decreasing. the model has a peak value within a certain range. It can be seen from the contour line of response surface that the contour line of response surface is close to ellipse, indicating that the interaction between the inoculation amount of bacteria and the proportion of enzymatic hydrolysate of ripened potato and pure milk is significant.
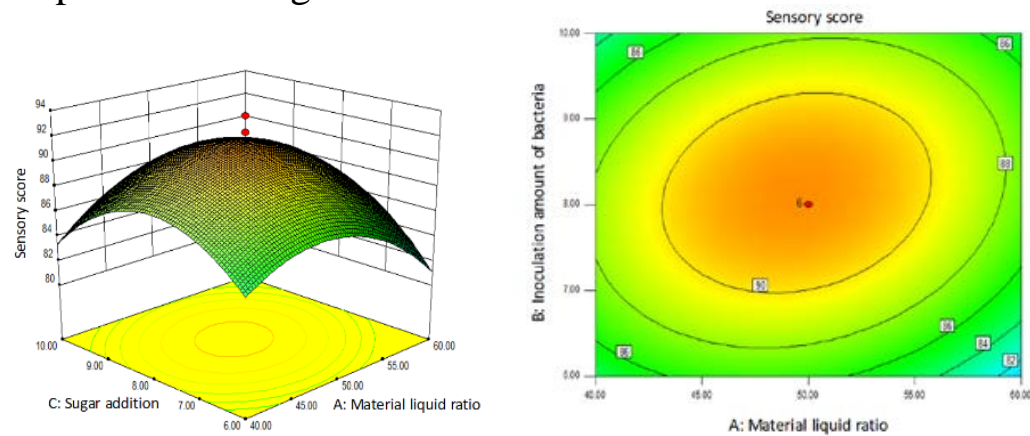

Figure 7 The interaction between the ratio of cooked potato enzymolysis solution and pure milk and the amount of cotton white sugar added

From fig. 7, it can be seen from the contour lines of the response surface that the contour lines of the response surface are close to oval, which indicates that the interaction between the sugar addition amount and the ratio of cooked potato enzymatic hydrolysate to pure milk is significant.
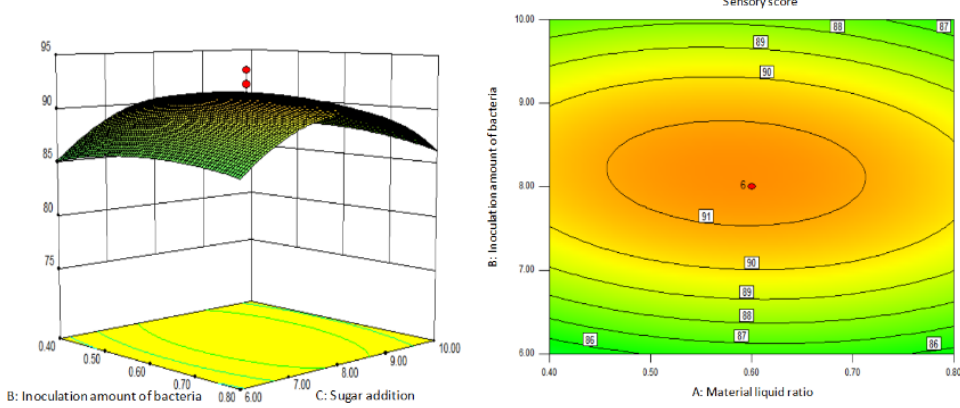

Figure 8 Interaction between the amount of starter inoculum and the amount of cotton white sugar added

As can be seen from fig. 8, as the inoculation amount of the strain and the addition amount of sugar gradually increase. From the contour lines of the response surface, it can be seen that the contour lines of the response surface are close to oval, which indicates that the interaction between the inoculum amount and the added amount of sugar is significant.

\subsubsection{Verification of fitting optimization}

Response surface analysis was used to optimize the technology of potato flavor yoghurt: the ratio of cooked potato enzymatic hydrolysate to pure milk was $1: 1$, the addition of starter was $0.6 \%$, the addition of soft sugar was $8 \%$, the fermentation time was $4 \mathrm{~h}$, and the fermentation temperature was $42^{\circ} \mathrm{C}$. According to the optimization plan, the average value of the sensory score of the product is 92.5, which is very close to the response value 92.0983 predicted by the model. It is verified that there is a good fit between the model and the real data.

\section{Discussion}

This experiment optimized the formula of potato flavor yogurt by response surface methodology, aiming to provide some theoretical reference for the development of potato fermented food. During the experiment, some problems were encountered in the storage of potato flavor yoghurt. The potato flavor yoghurt is easy to breed mold and yoghurt is easy to deteriorate. If this fermented food is really to be used in industrial production, its storability is still worthy of serious study. 


\section{References}

[1] Ruan Jun, Peng Guozhao, Luo Qing. Current status and countermeasures of potato industry development in Liangshan Prefecture [J]. Modern Agricultural Science and Technology, 2008(04): 54-56

[2] Xie Mingyong, Xiong Tao, Guan Qianqian. Research progress of key technologies for fermentation of fruits and vegetables with probiotics[J]. Chinese Journal of Food Science, 2014, 14(10): $1-9$

[3] Li Honggao. Development of stirred potato yogurt[J]. Food Science, 2007(07):597-600

[4] Xiong Zhengji, Wang Wenjia, Wen Rui, Dong Quan. Response surface method to optimize the fermentation process of inulin yogurt [J]. Food Science, 2014, 35(13): 156-160

[5] Nam Jo Kim,Hye Lim Jang,Kyung Young Yoon. Potato juice fermented with Lactobacillus casei as a probiotic functional beverage[J]. Food Science and Biotechnology,2012,21(5)

[6] Wu Jianmin, Liu Shaojun, Shao Songlin. Optimization of processing technology of potato yogurt[J]. Food and Machinery, 2015, 31(01): 222-225

[7] Shaobo Liang,Armando G. McDonald,Erik R. Coats. Lactic acid production with undefined mixed culture fermentation of potato peel waste[J]. Waste Management,2014,34(11) 